\title{
Des liens sociaux à la construction d'un développement territorial durable : quel rôle de la proximité dans ce processus?
}

Valérie Angeon, Patrick Caron et Sylvie Lardon

\section{OpenEdition}

1 Journals

Édition électronique

URL : http://journals.openedition.org/developpementdurable/2851

DOI : 10.4000/developpementdurable.2851

ISSN : 1772-9971

Éditeur

Association DD\&T

\section{Référence électronique}

Valérie Angeon, Patrick Caron et Sylvie Lardon, « Des liens sociaux à la construction d'un développement territorial durable : quel rôle de la proximité dans ce processus ? ", Développement durable et territoires [En ligne], Dossier 7 | 2006, mis en ligne le 17 juillet 2006, consulté le 10 décembre 2020. URL : http://journals.openedition.org/developpementdurable/2851 ; DOI : https://doi.org/ 10.4000/developpementdurable.2851

Ce document a été généré automatiquement le 10 décembre 2020.

\section{(c) (1) 8}

Développement Durable et Territoires est mis à disposition selon les termes de la licence Creative Commons Attribution - Pas d'Utilisation Commerciale 4.0 International. 


\title{
Des liens sociaux à la construction d'un développement territorial durable: quel rôle de la proximité dans ce processus?
}

\author{
Valérie Angeon, Patrick Caron et Sylvie Lardon
}

1 L'avènement des problématiques de développement durable (DD) amène à s'interroger sur plusieurs fronts : temporalité, équité, éthique, échelle spatiale, etc. Depuis quelques années, émerge un champ de réflexion fécond sur l'inscription territoriale du DD. Pour l'essentiel, deux voies de théorisation sont à considérer. Le lien entre DD et territoire renvoie, pour certains, à l'application des principes de durabilité à l'échelle locale. Dans ces conditions, le développement durable des territoires est une déclinaison locale des principes du DD'1. Pour d'autres auteurs, l'échelle territoriale permet d'appréhender de manière pertinente le socle du $\mathrm{DD}^{2}$ (Laganier, Villalba, Zuindeau, 2002). D'essence matérielle, identitaire et organisationnelle (Le Berre, 1995), le territoire est porteur de normes ou de règles produites par les acteurs locaux impliqués dans la définition de ses modalités de développement. Le développement territorial durable n'est autre qu'une construction d'acteurs visant une trajectoire particulière intégrant notamment l'évolution conjointe des systèmes économiques, sociaux et écologiques.

2 En nous inspirant de la définition du développement territorial - capacité des acteurs situés sur un territoire à en maîtriser les évolutions à venir (Deffontaines et al., 2001) -, nous convenons à l'idée que le développement territorial durable se définit comme la construction et l'articulation, par les acteurs locaux, de normes économiques, écologiques et éthiques. Il s'agit dès lors d'analyser la manière dont les groupes sociaux parviennent à édicter des règles et autres institutions facilitant l'élaboration de projets territoriaux communs. Tel est l'objet de notre article qui s'intéresse aux modalités de développement des territoires en soulignant l'importance des coordinations locales non marchandes dans ces processus.

3 Notre réflexion invite à une exploration théorique des mécanismes de développement des territoires dont il faut rappeler que la traduction dans l'agenda politique - tout au moins 
dans le contexte institutionnel français ${ }^{3}$ - appelle à une plus grande observation des principes de durabilité. Après avoir étudié la manière dont les coordinations locales influent sur les dynamiques de développement territorial, nous tenterons, dans la deuxième partie de ce texte, d'en conceptualiser les mécanismes. Nous retiendrons, pour ce faire, les approches du capital social (Lin, 1995, 2001) et de la proximité (Bellet et al., 1993 ; Bellet et al., 1998 ; Gilly et Torre, 2000 ; Pecqueur et Zimmerman, 2004). Dans la dernière partie de l'article, nous nous intéresserons à une caractérisation spatiale des liens sociaux. Nous mobiliserons les différents registres de la proximité pour qualifier la traduction spatiale des relations sociales et comprendre la manière dont elles évoluent dans la perspective d'un développement territorial durable.

4 Le développement territorial durable, en suscitant l'émergence d'un projet collectif, place les acteurs au cœur de la gouvernance des territoires. Il exprime la volonté de prendre en compte les besoins des populations locales et de répondre à leurs aspirations. Les intérêts des communautés locales s'expriment à travers la formalisation d'un projet territorial qui prend forme au sein d'une architecture institutionnelle particulière.

5 Tel qu'énoncé par les dispositifs institutionnels qui le consacrent, le développement territorial durable repose sur une double logique : l'établissement d'un projet de territoire associant les acteurs locaux à sa conception et le respect des principes de développement durable. La LOADDT ${ }^{4}$, la loi relative au renforcement et à la simplification de la coopération intercommunale ${ }^{5}$, la loi $\mathrm{SRU}^{6}$ (pour ne citer que les récentes modifications législatives) déterminent de nouvelles finalités pour l'action publique centrées sur les notions de sociabilité, de solidarité et de durabilité. Ces cadres institutionnels s'inscrivent dans la lignée des préconisations du sommet de Rio en 1992 qui conduit à la déclinaison à l'échelle territoriale d'engagements internationaux en matière de développement durable. Ces nouvelles formes de recomposition territoriale sont des lieux d'établissement de relations entre acteurs situés, ce qui leur vaut d'être assimilés à des espaces de solidarité et de proximité en vue d'un développement territorial durable ${ }^{7}$.

6 Ces structures nouvelles offrent de nouveaux référentiels pour l'action locale. D'une manière générale, dans ces dispositifs institutionnels, les centres d'intérêts collectivement exprimés par les acteurs sont inscrits dans des chartes. La charte repose sur l'existence d'un accord local (sur les principes et les finalités de l'action) indépendamment de toute référence au document formel matérialisant les engagements des partenaires. Ce qui importe ici, c'est le caractère conventionnel de ces chartes. L'engagement des acteurs est volontaire. La longévité des relations de partenariat esquissées dépend de la capacité des acteurs à adhérer à ses principes, à partager une même vision du territoire, une conception commune de ses modalités ou potentialités de développement.

7 Ces projets de territoire sont le support de démarches, stratégies ou initiatives visant à son développement. Ils définissent, à ce titre, des objectifs à atteindre sous certaines conditions ou contraintes. Un projet caractérise « la conjonction d'analyses, de désirs et de savoir-faire collectifs qui permet de polariser l'action de chacun autour d'une ambition commune, de résister aux forces centrifuges, de surmonter les contradictions internes d'intérêts, de saisir les opportunités qui se présentent d'exploiter les marges de manœuvre, de replacer l'action de chacun improvisée en fonction d'événements aléatoires dans une perspective à long terme » (Calame, 1991, p. 35). 
8 L'idée d'accord entre acteurs n'est pas sans poser de questions sur la manière dont les intérêts individuels sont rendus compatibles. La mise en cohérence des intérêts privés ne relève pas, en effet, d'une harmonie spontanée et l'expression des intérêts privés peut contrecarrer les logiques collectives. La propension des agents à s'entendre et à s'organiser - bref, à se coordonner - est ici cruciale.

8

9 Notre réflexion sur le développement territorial durable nous amène à nous intéresser à la dimension spatiale du développement. L'hypothèse avancée est que les dynamiques d'évolution différenciées que connaissent les territoires sont liées aux comportements des acteurs. Le faisceau de relations qu'entretiennent ces derniers, les réseaux qu'ils mobilisent, le poids du temps long avec ses implications en termes d'apprentissage collectif, importent dans l'analyse des trajectoires des territoires. En ce sens, le territoire est le résultat de jeux d'acteurs en relation les uns avec les autres. Ces éléments d'analyse nous invitent à mettre l'accent sur les coordinations locales, facteurs de développement. On s'intéressera dès lors à la nature de ces coordinations et aux effets qu'elles génèrent.

10 L'analyse montre que les relations entre acteurs, au-delà des systèmes d'intérêt et des jeux d'alliance et de concurrence qui en résultent, sont conditionnées par des systèmes de valeurs. Ces règles (entendues ici au sens large) normalisent leurs comportements. Elles ne sont pas nécessairement formalisées, elles peuvent être tacites. Ces règles partagées correspondent à un ensemble d' «institutions invisibles » qui facilitent les capacités d'anticipation des agents (i.e. ils éclairent les autres sur leurs intentions d'action) et régulent leur liberté de conduite et d'action. Favorisant une meilleure compréhension entre les agents, encourageant la transparence et la circulation d'informations, ces règles facilitent la coopération. Elles permettent, en outre, de déboucher sur des régularités de comportement et préviennent les défections ou les comportements opportunistes. Elles contribuent ainsi à stabiliser ou à renforcer les liens entre les agents en favorisant le développement de signes qui limitent les problèmes d'asymétrie d'information, d'incertitude et les dissensions.

11 Soutenir que les coordinations locales non marchandes ont une incidence sur le développement implique d'identifier les mécanismes auxquels elles renvoient. Certains économistes proposent une grille d'analyse de ces mécanismes appréhendés comme des externalités. On démontre alors que les coordinations locales génèrent des externalités positives. On en distingue deux types. Le premier se rattache à des questions de collecte et de circulation de l'information (1.2.1.). Le second se rapporte à l'action collective (1.2.2.).

12 Les exemples sont nombreux, dans les cas de collaboration possibles ou souhaités, où les agents sont conduits à prendre des décisions inefficaces par manque d'informations. Les cas des marchés du travail et du crédit peuvent être cités à ce titre. Ils illustrent des situations d'incomplétude ou d'asymétrie d'information formalisées notamment par la théorie de l'agence. Comme nous l'avons exposé précédemment, les liens qu'entretiennent les agents reposent sur un ensemble de règles qui facilitent la coordination entre eux. Elles favorisent non seulement une meilleure connaissance des comportements individuels mais permettent également un accès plus facile (et moins coûteux) à des données concernant leur environnement immédiat.

13 À ce titre, l'exemple du progrès technique dans le processus de production des entreprises peut être donné. Il met en évidence la qualité des coordinations locales dans 
le partage et la diffusion de l'information. Ces informations, qu'elles concernent la transmission d'une technique de production ou d'un apprentissage (savoir-faire), sont sources de compétitivité pour les entreprises. L'accès de ces firmes à de telles informations se solde par une meilleure connaissance de leur environnement économique. Les districts industriels et les systèmes productifs locaux fonctionnent sur ce mode. Ils décrivent des situations où le degré d'interaction entre des agents appartenant au même milieu socio-culturel et ratifiant les mêmes normes de comportement - est élevé.

En somme, en situation de coopération, le partage de l'information améliore la qualité des décisions par rapport à des procédures individuelles de choix. La meilleure circulation de l'information nouvelle ou le partage d'un capital cognitif que favorisent les liens locaux s'avèrent dès lors avantageux dans la mesure où ils contribuent à réduire le risque, l'incertitude et à économiser les coûts de transaction.

Dans les projets de territoire, le principe de gouvernance locale rend bien compte d'une certaine efficience collective. Les agents, associés à la prise de décision, s'organisent sur le mode de la coopération. Amenés à partager des informations de diverse nature, ils détiennent une meilleure connaissance des individus et de leurs intentions ainsi que de leur environnement socio-économique. Par ce biais, ils améliorent la qualité de leurs choix, ce qui leur permet de prendre véritablement part au dispositif d'action et d'augmenter leur degré de maîtrise du territoire.

Au-delà de la seule transmission de l'information, les processus de construction territoriale relèvent du partage d'informations. Ils s'inscrivent dans une dynamique collective d'apprentissage visant à l'élaboration d'un socle commun de connaissances, d'une culture commune. Cette accumulation de connaissances, résultat d'un construit social, facilite et renforce les stratégies d'action collective, au cœur des logiques de développement territorial.

17 Le second type d'externalité que génèrent les coordinations locales est l'action collective. Le principe de l'action collective repose sur l'habileté des agents à mettre en commun des ressources pour atteindre des objectifs qui n'auraient pas été atteints individuellement. On convient ainsi à l'idée que l'action collective résulte des dynamiques de coopération qui s'établissent entre les agents.

L'étude des mécanismes coopératifs a fait l'objet de nombreuses analyses en économie. Principalement formalisé par la théorie des jeux, le problème de la coopération se pose lorsque l'on cherche à expliquer comment les individus agissent en fonction d'un objectif commun, alors qu'a priori selon leur strict intérêt personnel, ils ne gagnent pas à entreprendre cette action.

19 L'analyse montre qu'en situation de jeu répété, les agents sont susceptibles de développer un comportement coopératif. Si l'on se fie à l'observation des comportements effectifs des individus, on constate que la coopération résulte d'une combinaison de règles (valeurs, normes conventions, routines, etc.). De manière plus intuitive, on se range à l'idée que l'occurrence des interactions amène les individus à développer des routines. Ces routines stabilisent les dynamiques d'interaction parce qu'elles permettent aux agents d'acquérir des informations essentielles visant à mieux comprendre et à anticiper leurs intentions réciproques d'action. Elles s'accompagnent d'autres phénomènes (i.e. la réputation, confiance, réciprocité) qui confortent l'espace relationnel. Les relations que 
nous décrivons ne sont pas exclusivement bilatérales, les agents entretenant en effet une pluralité de liens.

Comme nous venons de l'évoquer, les comportements coopératifs demeurent au fondement de l'action collective. Ils mettent en évidence l'ensemble des relations interpersonnelles dans lesquelles s'inscrivent les agents. Dans cet espace de proximité que constitue le territoire, les institutions invisibles qui procèdent à la coordination des agents placent ces derniers en situation de développer des liens s'apparentant à ceux que l'on observe dans des structures de type communautaire. Ces réseaux communautaires qui reposent sur une nature de liens particuliers entre agents (de solidarité, de coopération, de proximité), imposent dès lors une représentation complexe du territoire et de ses modalités de développement.

Comme le souligne B. Zuindeau (2000), le DD repose sur un projet et non sur l'application d'un modèle abouti, constant et invariable. En cela, le développement durable d'un territoire est contingent aux singularités de ce territoire. Ces dernières tiennent à la fois dans les aspects matériels, idéels ou organisationnels du territoire. Elles laissent place, ce faisant, à l'interprétation des acteurs, à leur intentionnalité, à leur capacité à produire des arrangements territoriaux. Le développement territorial durable peut alors désigner une dynamique d'initiatives locales qui met en mouvement des acteurs (privés et/ou publics) à travers des formes de relations sociales organisées. Il reste à appréhender du point de vue théorique ces dynamiques de relations sociales. Les approches du capital social et de la proximité constituent des cadres d'analyse appropriés à ces questions.

Les deux approches ont en commun de livrer un éclairage sur les mécanismes de développement territorial (Angeon et Callois, 2004b). L'approche du capital social appréhende, à travers l'analyse des liens sociaux (nature, densité), les différentes ressources mobilisables pour le développement local. L'économie de proximités, quant à elle, fournit un cadre d'analyse des différents modes d'interaction entre acteurs situés sur un territoire.

23 Le concept de capital social n'est pas récent ${ }^{9}$. Mis en avant par Coleman $(1988,1990)$, dans sa théorie de l'action rationnelle, le terme renvoie aux déterminants sociaux qui rendent possible l'action. Nous présentons ci-après les principales propositions de Coleman sans prétention d'exhaustivité. Pour Coleman, le capital social se définit par sa fonction (faciliter l'action des individus). Il existe sous trois formes : (i) les obligations et attentes réciproques dans une unité sociale, (ii) la crédibilité de l'information véhiculée par le biais des relations sociales, (iii) les normes et sanctions effectives facilitant le contrôle social et évitant de recourir à des procédures ou outils formels.

Cette vision fonctionnaliste amène à caractériser le capital social par ses effets. Dès lors, on ne s'intéresse pas à sa nature intrinsèque. Cette démarche d'analyse est problématique à plus d'un titre. Elle conduit à surdéterminer l'impact du capital social. Elle tend à confondre la cause et les effets du capital social. On notera, par exemple, que deux des formes de capital social identifiées par Coleman (obligations réciproques et normes) ne posent pas ce dernier au fondement des relations sociales mais le présentent au contraire comme un dérivé de la structure sociale. La conception fonctionnaliste du capital social tend à obscurcir la notion, la présentant à la fois comme un produit de la structure sociale et comme un élément structurant qui la détermine. Par ailleurs, elle conduit à des raisonnements circulaires et tautologiques. On peut évoquer par exemple, le truisme fréquemment cité des sociétés prospères décrites par la performance de leur structure sociale - i.e. leur degré élevé de capital social - et inversement (Lin, 1995 ; Portes et 
Landolt 1996 ; Portes, 1998 ; Fukuyama, 1999 ; Woolcok, 2001). Ce raisonnement inductif, par essence, présume de l'existence de capital social en présence de ses effets supposés.

Se centrant davantage sur les forces du capital social que sur ses formes ${ }^{10}$, Sandefur et Laumann (1998) retiennent trois de ses caractéristiques fondamentales. (i) Le capital social peut procurer - tout comme chez Coleman - des avantages en termes d'information, d'influence et de contrôle, de liens de solidarité. Ils admettent par contre (ii) que le capital social facilite l'obtention d'un ou plusieurs objectifs et (iii) génère, à certains égards, des effets positifs ou négatifs pouvant par conséquent entraver la réalisation d'objectifs concurrents. À ce titre, Portes et Landolt (1996) mettent en évidence plusieurs aspects négatifs du capital social. Engoncés ou contraints par leur tissu relationnel, les agents peuvent au final opter pour des stratégies ou des comportements non optimaux. Ces derniers sont le résultat de plusieurs facteurs. Callois (2004) en dénombre trois types : les difficultés d'accès à des informations nouvelles et diversifiées, le manque d'incitation ou de motivation individuelle et la tendance à la discrimination ou la collusion nuisant à l'esprit de coopération et portant préjudice à l'action collective. Ces aspects négatifs du capital social affectent le bien-être social.

Bien que la démarche de Sandefur et Laumann soit intéressante, par sa considération plurielle des effets des relations sociales, elle s'inscrit également dans une approche fonctionnaliste. Elle étend le raisonnement de Coleman sur les fonctions et les formes de capital social à un examen de ses seules conséquences. Elle proroge la tradition de définition du capital social par ses effets plutôt que par ses attributs propres.

La seconde voie de définition du capital social se penche sur sa nature et vise à dissocier ses propriétés intrinsèques de ses effets. Elle caractérise le capital social comme une ressource confinée dans des pratiques de sociabilité. Le capital social est ainsi une ressource sociale produite et valorisée au sein d'un réseau social. Parmi les travaux qui associent le capital social à une ressource réticulaire, figurent ceux de Bourdieu (1980, 1986) et de Lin (1995).

Suggérant d'intégrer le capital social dans une théorie générale des ressources, Lin (1995, 2001) le définit comme une richesse potentielle incorporée dans la structure sociale et qui peut être (mais ne l'est pas nécessairement) mobilisée en cas de besoin. Le concept de capital social recouvrirait ainsi à la fois des ressources existantes et latentes liées à un réseau relationnel stable plus ou moins activé. Le capital social est « l'investissement d'un individu dans ses relations avec d'autres » (Lin, 1995, p. 701). Il devient dès lors possible d'identifier les lieux de production du capital social et partant, de procéder à leur caractérisation indépendamment de leurs effets.

Cette conception rejoint celle adoptée par Bourdieu pour lequel « le capital social que possède un agent particulier dépend de l'étendue du réseau des liaisons qu'il peut effectivement mobiliser et du volume du capital (économique, culturel ou symbolique) possédé en propre par chacun de ceux auquel il est lié ». On rappellera que le capital social est une ressource dont disposent les agents mais que ce n'est pas la seule. En outre, l'individu peut être inséré dans un tissu relationnel dense (stock élevé de capital social) sans que cela ne lui soit directement profitable. Les deux conditions essentielles à l'existence de capital social tiennent dans le caractère fongible des formes de capitaux mais aussi dans la disponibilité des ressources (autres que sociales) inscrites dans les relations réticulaires. 

d'exemple, lorsqu'une aide financière est proposée à un individu par un membre de son réseau relationnel, le capital social se transforme en capital économique. De la même façon, lorsqu'un individu se sert de son réseau pour accéder à une formation, il tend à améliorer son niveau de qualification initiale. Le capital social se trouve ainsi transformé en capital humain. Le capital social est interdépendant des autres formes de capital. Il peut être, pour reprendre les termes d'Adler et Kon (1999), un substitut ou un complément à d'autres ressources ${ }^{11}$.

31 Un autre élément important de la cohérence du raisonnement bourdieusien relève de la disponibilité des ressources contenues dans les relations sociales. Autrement dit, il doit exister un lien social mais celui-ci doit être pourvoyeur de ressources ${ }^{12}$. En outre, si toutes les relations possèdent un potentiel de capital, l'activation du capital latent est fonction de la nature des relations qu'entretiennent les agents. En clair, le bénéfice tiré par un agent de son investissement social dépend fortement de la reconnaissance qu'en ont les autres ${ }^{13}$. Ainsi, le capital social n'est autre que «l'ensemble des ressources actuelles ou potentielles qui sont liées à la possession d'un réseau durable de relations plus ou moins institutionnalisées d'interconnaissance et d'inter-reconnaissance » (Bourdieu, 1980, p. 2.).

Les approches de Lin et de Bourdieu présentent une certaine filiation. Ces conceptions réticulaires se distinguent de l'approche fonctionnaliste initiée par Coleman à laquelle on reproche de ne pas être en mesure de saisir les caractéristiques propres du capital social. Cela conduit à présenter comme supérieure la validité heuristique des théorisations d'essence relationnelle du capital social (Lévesque, 2000). Cette vision dynamique des relations sociales invite à se pencher sur la nature des liens tissés entre les agents. La Banque mondiale a, par exemple, proposé une typologie des liens sociaux en 2000 ; elle qualifie les liens sociaux en termes de "bonding", «linking", "bridging" (respectivement capital social qui unit, lie et relie).

Les liens de type "bonding " unissent des individus au sein d'une même communauté. Ces liens sont encore qualifiés parfois d'horizontaux. Dans ce type de structure sociale, les agents sont de statut identique : ils appartiennent au même groupe. On y range les relations familiales et amicales, les réseaux sociaux de type communautaire, les organisations, etc. Les liens de type «linking " caractérisent des interactions entre des agents appartenant à des groupes différents. Ces liens sont également dits verticaux. Dans ce type de relation, les agents occupent des places (ou des statuts) différents. L'exemple le plus couramment cité est celui de la coopération interfirme (i.e. les entreprises dans les districts industriels ou les systèmes productifs locaux au titre de leurs relations complexes de concurrence, de sous-traitance mais aussi d'entraide). Enfin, dans les relations de type "bridging", les agents sont distants les uns des autres. Cette dernière notion désigne à la fois leur éloignement physique (les liens de type bridging sont extraterritoriaux) et l'absence ou la discontinuité dans l'activation du lien (l'idée de bridging rend compte du caractère latent du capital social, apparenté dans ce cas à une ressource non activée). Le «bridging social capital» désigne un réseau virtuel mais potentiel. Il caractérise des acteurs qui interviennent dans des espaces d'action distincts et ratifient des normes ou des valeurs différentes.

Cette synthèse sur la nature des liens peut être rapprochée de la vision dichotomique de Granovetter (1973), distinguant les liens forts des liens faibles. Le capital social de type bonding et linking peut être saisi comme un ensemble de liens forts, alors que le capital social de type bridging appartient à la catégorie des liens faibles. Ces considérations de la

Développement durable et territoires, Dossier 7| 2006 
structure sociale s'expriment en termes de proximité ou, au contraire, de distance sociale.

Le concept de proximité renvoie à une dénomination plurielle (Bellet et al., 1998; Rallet, 2002). Le terme est d'un usage courant et recouvre diverses notions. On parle en effet de relations de proximité, d'emplois de proximité, de services de proximité, de justice de proximité, etc. Par ailleurs, la proximité se décline selon divers registres ou ordres. Les termes de proximité géographique, organisationnelle, relationnelle, matérielle, territoriale, institutionnelle, etc., se côtoient dans la littérature mais les principaux contributeurs s'accordent à retenir deux déclinaisons analytiques de la proximité : (i) la proximité géographique et (ii) la proximité organisée. Ces composantes de la proximité expriment respectivement la séparation géographique et économique des agents.

36 La proximité organisée traite de la séparation économique des agents. Elle identifie l'espace de rapports des individus. De ce point de vue, sont proches des acteurs qui appartiennent au même espace de rapports et entre lesquels s'établissent des interactions de nature différente (marchandes ou non marchandes, intentionnelles ou non intentionnelles, etc.). La proximité organisée renvoie également à l'espace de représentation des individus. Selon cette dernière acception, sont désignés proches des agents qui se ressemblent et qui partagent le même espace de valeurs, de règles, de modèles de pensées et d'actions.

En clair, la proximité organisée repose sur deux logiques ou recouvre deux dimensions : la logique d'appartenance et la logique de similitude. Dans la première dimension, c'est l'effectivité des relations qui est évoquée, alors que dans la seconde, c'est de l'adhésion à un même espace de référence dont il est question. Cette deuxième dimension de la proximité organisée (la logique de similitude) relève du domaine institutionnel. Elle est appelée par certains auteurs proximité institutionnelle et est considérée parfois comme un registre à part de la proximité.

La proximité géographique décrit la position relative des agents dans un espace plan déterminé. Elle renvoie à la localisation des interactions entre les agents au sein d'un espace précis. Elle rend compte de leur éloignement, et se mesure en termes de distance exprimée en fonction du temps et/ ou des coûts à supporter pour la franchir. Dans ces conditions, on conçoit que la proximité géographique n'est pas strictement naturelle ou physique, l'espace physique étant anthropisé. Elle est à la fois support et construit d'interactions sociales. Cette forme de proximité fait référence à la notion d' « espace géonomique » de Perroux (1964), "renvoyant largement à la localisation des entreprises, elle intègre la dimension sociale des mécanismes économiques, ou ce que l'on appelle parfois la distance fonctionnelle» (Zimmermann et al., 1998, p. 9).

«La proximité géographique traduit la plus ou moins forte matérialité de l'espace : elle est traitée comme une contrainte pesant sur le développement des interactions entre agents. Dans l'analyse, elle joue le rôle d'une condition permissive : la proximité favorise a priori le développement des interactions entre agents. Elle facilite les échanges de produits mais aussi les rencontres, les échanges d'information, le partage des connaissances » (Rallet, 2002, p. 13). Selon cette assertion, la proximité géographique favoriserait l'établissement de liens entre les agents. Elle permettrait le développement de liens de type organisationnel. On peut aussi associer à la proximité géographique lorsqu'elle se circonscrit à un espace précisément délimité et stable - à un lieu commun de représentations au sein duquel on identifie des aspects tels que la culture, les 
traditions, les valeurs, etc. Comprise en ce sens, la proximité géographique pourrait expliquer certaines ressemblances entre les agents en termes de représentation. Elle favoriserait ainsi la dimension institutionnelle de la proximité organisée. Par extension, elle recouvre la notion de proximité géographique de configuration (Moquay et al., 2005) ${ }^{14}$, qui ne repose pas sur la localisation, mais sur l'existence de propriétés communes de l'espace auxquelles les agents se réfèrent pour agir ${ }^{15}$.

40 À l'issue de ces propos, on convient du rôle de la proximité géographique dans la constitution des relations de face à face d'une part, et dans la diffusion de règles et de modèles d'actions communs d'autre part. En d'autres termes, la proximité géographique peut favoriser le renforcement des logiques d'appartenance et de similitude qui caractérisent la proximité organisée. Mais elle peut être aussi génératrice de conflits (Torre et Caron, 2002). Comme en attestent les propos précédents, les deux types de proximité s'articulent.

41 L'économie de proximités et l'approche du capital social présentent des démarches d'analyse complémentaires. Elles abordent la nature des liens tissés entre les acteurs et visent à caractériser leurs modalités de coordination. Nous verrons, par la suite, que la procédure d'activation des liens sociaux repose sur des logiques de proximité.

42 Les travaux sur le capital social montrent qu'il est susceptible d'évoluer dans le temps (le stock de capital n'est pas invariant). Il est fonction de la capacité des agents à tirer parti de leurs réseaux sociaux. L'accumulation de capital social s'effectue à travers la mobilisation des relations sociales. En somme, la productivité du réseau (en termes de ressources sociales) ne dépend pas que du lien établi entre des agents ou groupes d'agents mais de l'activation de ce lien. Autrement dit, la création et l'accumulation de capital social impliquent que les liens entre individus soient mobilisables et mobilisés.

43 L'analogie entre le capital social et les autres formes de capital réside dans le fait qu'il peut être à la fois acte de consommation et d'investissement. Par exemple, lorsqu'un agent active un lien social, en recourant au service d'une connaissance, il consomme du capital social. Mais en même temps, comme il entretient la relation, il procède également à un investissement social. Le demandeur de service est placé dans une relation d'obligation et de dette par rapport à l'offreur. Ce dernier, lorsqu'il consent à rendre service témoigne une preuve de confiance au bénéficiaire. Cette interaction sociale augmente la force du lien entre les deux individus insérés dans la relation en générant de nouvelles règles de rapport. Ce faisant, cette interaction sociale contribue à augmenter le capital social.

44 Ces éléments d'analyse nous invitent à souligner la particularité du capital social par rapport au capital physique. À l'inverse de ce dernier, le capital social ne se déprécie pas avec l'usage. Bien au contraire, il se délite lorsque les liens sociaux ne sont pas entretenus ${ }^{16}$. En conséquence, l'accumulation de capital social dépend étroitement non seulement de la qualité des relations entre les agents mais également de leur régularité. Les liens sociaux doivent être continuellement réactivés, au risque de perdre en efficacité. Dans cette conception, le capital social pourrait renvoyer à des considérations patrimoniales au sens défini par B. Billaudot (2004) car il ne rend pas compte d'un processus de destruction et d'appropriation mais se constitue sur le mode de la conservation et de la transmission. Cette définition est inspirée des travaux de Barrère et al. (2001).

45 Si l'on rapporte cette dernière conclusion à notre caractérisation des liens sociaux, on admet que les liens sociaux de type bonding ou linking nécessitent d'être constamment 
renforcés pour que le capital social soit effectivement une ressource utile. En revanche, le capital social de type bridging n'implique pas une activation régulière, ni intense des liens. Il faut à ce titre observer la spécificité de cette forme qui repose par définition sur l'établissement de liens faibles. En clair, le capital social, pour être efficacement mobilisé, doit s'appuyer sur une dynamique de liens sociaux particulière. Il repose sur une certaine densité de rapports, ce qui suppose que les agents s'inscrivent dans des relations de proximité. Ces structures relationnelles sont plus ou moins denses et cohésives.

La nature des liens élaborés entre les agents peut ainsi être caractérisée par leur densité et appréhendée en termes de proximité. Le tableau 1 ci-dessous synthétise notre propos.

Tableau 1 Nature et densité des liens

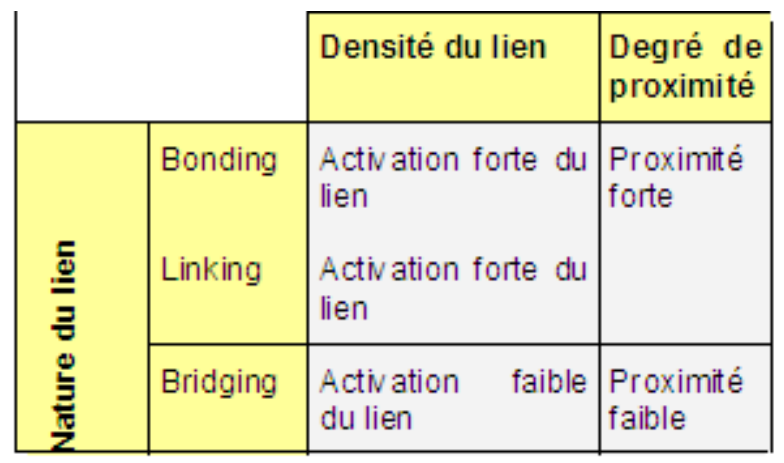

Nous avons souligné précédemment que le capital social doit être entretenu à travers l'activation de liens sociaux. Une bonne compréhension de la procédure d'activation des liens nous invite à l'analyse des règles qui président aux relations entre agents. L'approche en termes de proximité permet de spécifier ces règles.

Les liens de type bonding caractérisent des relations affectives, des affinités sociales. Ces liens sont de type communautaire et sont porteurs d'une logique d'exclusion des membres extérieurs à la communauté. Ils tendent vers une "fermeture relationnelle " (Coleman, 1988). L'appartenance à une même communauté rend compte de ce que les agents adhèrent au même système de représentation. Et dans ce type de liens, la confiance interpersonnelle est élevée. Les agents élaborent leurs propres règles et organisent leur contrôle. Ces liens intra-groupe expriment un degré de proximité fort entre les agents. Ici, c'est le registre institutionnel de la proximité qui prévaut (logique de similitude) mais il n'exclut pas la dimension organisationnelle (logique d'appartenance).

Le capital social de type linking nécessite une activation régulière des liens sociaux. Ces liens inter-groupes doivent par conséquent être continuellement réaffirmés. Le processus d'échange entre les agents implique la fréquence des interactions. Il se caractérise par des transactions de réciprocité qui obligent à la poursuite des échanges. C'est bien l'effectivité des relations qui est ici soulignée. Elle fait référence au registre organisationnel de la proximité (logique d'appartenance). Comme nous l'avons déjà mentionné, la fréquence des interactions tend à déboucher sur la convergence des représentations (élaboration de normes communes, réciprocité) et peut ainsi nourrir la dimension institutionnelle de la proximité. Dans le capital social de type linking, c'est donc d'abord le registre organisationnel de la proximité qui est au fondement des relations mais il n'est pas exclu que la logique institutionnelle de la proximité contribue à les renforcer. 
50 Ainsi, si les liens de type bonding et linking reposent sur des structures relationnelles cohésives qui créent et augmentent le sentiment d'appartenance et de solidarité entre les membres du groupe, dans les liens de type bridging, prévalent d'autres logiques. La fréquence des contacts n'est pas une condition nécessaire à la mobilisation du capital social. La procédure de mise en accord entre les agents est ponctuelle et discontinue. Les règles respectées s'apparentent alors à une convention sans engagement réciproque de leur part. Elles s'appuient cependant sur la reconnaissance temporaire d'une communauté d'intérêt qui justifie l'activation d'une relation inhabituelle. Dans ce cas, ce ne sont pas des logiques de proximité - telles qu'entendues par l'économie de proximités - qui œuvrent dans ce type de relation, mais ce que nous appelons logique de relation à distance. Cette logique a à voir avec la proximité géographique de configuration évoquée antérieurement. Le tableau 2 résume l'ensemble de ces propos.

Tableau 2 Nature des liens sociaux et logiques de proximité

\begin{tabular}{|c|c|c|c|}
\hline $\begin{array}{l}\text { Nature } \\
\text { du lien }\end{array}$ & $\begin{array}{l}\text { Motivation } \\
\text { du lien }\end{array}$ & $\begin{array}{l}\text { Nature et } \\
\text { propriétés } \\
\text { des règles } \\
\text { ratifiées }\end{array}$ & $\begin{array}{l}\text { Logique de } \\
\text { proximité } \\
\text { prévalant }\end{array}$ \\
\hline Bonding & $\begin{array}{l}\text { Morale } \\
\text { Investisse } \\
\text { ment social } \\
\text { non } \\
\text { intentionne } \\
\text { I }\end{array}$ & $\begin{array}{l}\text { Introjection } \\
\text { de valeur }\end{array}$ & $\begin{array}{l}\text { Logique de } \\
\text { similitude } \\
\text { (mais } \\
\text { n'exclut pas } \\
\text { la logique } \\
\text { d'appartena } \\
\text { nce) }\end{array}$ \\
\hline Linking & $\begin{array}{l}\text { Intérêt } \\
\text { Investisse } \\
\text { ment social } \\
\text { intentionne } \\
\text { I }\end{array}$ & $\begin{array}{l}\text { Réciprocité } \\
\text { et obligation }\end{array}$ & $\begin{array}{l}\text { Logique } \\
\text { d'appartena } \\
\text { nce } \\
\text { (mais } \\
\text { n'exclut pas } \\
\text { la logique } \\
\text { de } \\
\text { similitude) }\end{array}$ \\
\hline Bridging & $\begin{array}{l}\text { Intérêt } \\
\text { Investisse } \\
\text { ment social } \\
\text { intentionne } \\
\text { I }\end{array}$ & \begin{tabular}{|l|} 
Convention \\
sans \\
engagemen \\
t réciproque
\end{tabular} & $\begin{array}{l}\text { Logique de } \\
\text { relation à } \\
\text { distance } \\
\text { (Logiques } \\
\text { de } \\
\text { similitude et } \\
\text { d'appartena } \\
\text { nce } \\
\text { inexistantes } \\
\text { ) }\end{array}$ \\
\hline
\end{tabular}

Source : D'après Angeon et Callois, 2004b.

51 Notre analyse montre la contribution des différentes formes de proximités à la caractérisation du capital social. C'est à une relecture spatiale des formes de relations sociales que nous nous livrerons maintenant. Ceci nous permet de fournir un cadre conceptuel pour saisir la pluralité des configurations territoriales et leur interaction avec le capital social dans les processus de développement durable des territoires.

Le capital social est considéré comme un potentiel activable favorisant le développement durable des territoires. Pour appréhender les interactions entre capital social et développement territorial, nous proposons de considérer les formes d'organisation territoriale, entendue comme l'articulation des organisations sociale, spatiale et politique 
(Lardon et al., 2003). Nous avons croisé les formes de réseaux sociaux et les registres de proximité qui animent les liens sociaux (cf. partie 2). Il nous reste à qualifier spatialement ces structures sociales, dans le cadre des politiques et des projets territoriaux, s'inscrivant entre incitation publique et initiative locale. Nous tenterons alors d'analyser les relations sociales mobilisant les différentes formes de proximité, en intégrant la dimension spatiale. Nous appréhenderons ces relations sociales en termes de configurations spatiales (3.1.). Cela nous conduira à repérer l'ensemble des formes de relations sociales visant, pour les groupes sociaux d'un territoire donné, à tenter d'en maîtriser les évolutions à venir. On explicitera dès lors comment la structuration sociale, en s'appuyant sur les configurations spatiales (3.2.), façonne les projets de développement durable des territoires. Nous appuierons notre réflexion sur les résultats d'un programme de recherche financé par le MEDD et le PUCA (septembre 2003 - juillet 2005).

Pour qualifier en termes spatiaux les liens sociaux, on se réfère aux configurations spatiales, dans la mesure où elles sont à la fois matérielles et idéelles. Elles sont liées à des pratiques et des projets et proposent, par le recours à un langage iconographique, une lecture du territoire (Debarbieux, 2003). Elles permettent de décrire la délimitation des réseaux sociaux et la manière dont ils sont inscrits dans l'espace, en s'appuyant sur les principes organisateurs de l'espace (Piveteau et Lardon, 2002). Elles renvoient ainsi à des formes d'organisation territoriale dont on peut expliciter la logique de construction.

Le croisement entre formes de capital social et logiques de proximité nous permet de retenir ici des cas de figure représentatifs de formes d'organisation territoriale.

- Les liens de type bonding sont marqués par une préexistence de la logique de similitude. Cependant, ils peuvent également être fortement activés (logique d'appartenance). Dans ce cas, la capacité d'organisation s'appuie sur les liens d'affection. La forme spatiale du réseau déclinée selon une logique de similitude coïncide par conséquent avec le périmètre $\mathrm{du}$ réseau décrit selon une logique d'appartenance. Ils ont le même pourtour, existent dans la même temporalité. Ces formes d'organisation se matérialisent dans le territoire et sont observables à travers la densité des relations au sein du périmètre considéré. Selon les territoires, ces formes d'organisation peuvent constituer des entités de taille différente. On peut citer, par exemple, l'échelle du hameau ou de l'intercommunalité comme échelle de sociabilité. Si l'on raisonne sur le temps long, on peut penser que l'intensification des relations (i.e. de la logique d'appartenance) renforce encore la logique de similitude.

- Les liens de type linking se caractérisent, à l'inverse, par la prévalence de la logique d'appartenance. Cette intensité de liens est susceptible d'enclencher la création de liens institutionnels (logique de similitude) et de les renforcer. Ce faisant, les liens de proximité institutionnelle confortent la proximité organisationnelle. Les deux logiques coexistent alors et se renforcent mutuellement. Spatialement, le périmètre d'action du réseau construit sur des logiques d'appartenance ne coïncide pas nécessairement avec celui du réseau constitué sur des logiques de similitude. La logique de similitude dépasse le périmètre d'action du réseau fonctionnant sur un mode organisationnel. Citons à titre d'exemple deux associations - animées par un objectif similaire - décidant d'agir ensemble. Ce faisant, elles peuvent se reconnaître un ensemble de valeurs communes de portée plus générale. Le périmètre organisationnel est alors inclus dans le périmètre institutionnel. Dans une vision de long terme, on peut considérer que la logique de similitude intensifie la logique d'appartenance et étend le périmètre d'action du réseau. Les espaces d'actions sont alors les mêmes ou du même ordre que les espaces de relations. 
- Dans le cas des liens de type bridging - pour lesquels n'existent a priori aucune logique classique de proximité - la logique de relation à distance ne repose pas sur un ensemble de liens sociaux effectifs. L'organisation réticulaire demeure donc virtuelle, mais elle reste possible. De même, la référence institutionnelle n'est pas explicite. Cependant, en cas d'activation temporaire, les logiques d'appartenance et de similitude peuvent s'activer de manière synchrone et ne perdurer que le temps de la relation. Ce faisant, elles peuvent impulser, au niveau du territoire, un renforcement des logiques d'appartenance et de similitude dans les réseaux existants et générer une nouvelle forme d'organisation. Ceci est vrai par exemple lorsque l'activation d'un lien extérieur au territoire - comme avec un financeur qui reconnaît la valeur du projet - consolide les actions menées localement (proximité organisationnelle), en renforçant l'esprit de cohésion (proximité institutionnelle) par la création d'une nouvelle entité. (...) Elles naissent de processus interactifs et sont alors engendrées dans leurs configurations. (...) Elles constituent l'expression du processus cognitif qui est engagé lorsque les acteurs ayant des compétences différentes produisent des connaissances nouvelles par la mise en commun de ces dernières " (Colletis et Pecqueur, 2004, pp. 4-5). Ainsi, des associations localisées, des villages avec une même morphologie de périurbanisation, un espace protégé... constituent autant de points d'ancrage potentiels à l'émergence de configurations territoriales porteuses de développement. En conséquence, notre approche spatiale des relations sociales nous amène à comprendre comment se crée et se renforce le capital social sur un territoire. On cherche à identifier les ressorts territoriaux qui facilitent l'émergence de formes d'organisation territoriale. la mobilisation ou l'activation de liens sociaux. Pour cela, nous considérons le capital social comme un ensemble de ressources potentielles (historiques, culturelles, patrimoniales, etc.), territorialement ancrées et spatialement matérialisées. On admettra également que le développement territorial consiste en l'activation de ces ressources et que ce potentiel relationnel et sa traduction en termes de capital social sont évolutifs et transformables. Nous appuierons notre propos sur une analyse empirique (Angeon et Callois, 2005). Cette étude visait à appréhender, dans des territoires de projets (pays au sens de la LOADDT), la 
structuration sociale et à caractériser son impact sur le développement durable des territoires. Nous avons considéré des territoires différenciés selon un double critère : leur niveau de développement économique et leur degré de capital social.

Pour évaluer a priori le niveau de développement d'un territoire, nous avons tenu compte des indicateurs de potentiel fiscal et de la variation de l'emploi entre 1990 et $1999^{17}$. Pour évaluer au préalable la propension à l'action collective dans ces terrains, nous nous sommes référés à des dires d'experts.

61 Nous avons ensuite mené deux séries d'enquêtes auprès d'institutionnels (élus locaux, chambres consulaires, responsables d'associations...) et d'habitants ${ }^{18}$. Nous avons retenu quatre territoires pour notre étude présentant des caractéristiques contrastées : le pays de Saint-Flour/Haute Auvergne (Cantal), le pays de Dinan (Côtes-d'Armor), le pays de Loire-Beauce (Loiret) et le pays du Forez (Loire). Suivant ces critères, le pays du Forez et le pays de Loire-Beauce présentent un niveau de développement élevé. Contrairement à ces derniers, les pays de Dinan et de Saint-Flour connaissent un développement que l'on peut qualifier de faible. En termes de capital social, le pays de Dinan et le pays du Forez présentent des dynamiques sociales élevées, contrairement aux pays de Saint-Flour et de Loire-Beauce où des logiques individualistes sont décrites comme prédominantes. Cette caractérisation des territoires est reprise dans le tableau 4 suivant.

Tableau 3. Caractéristiques des territoires étudiés

\begin{tabular}{|c|c|c|c|}
\hline & & Niveau de & $\begin{array}{l}\text { Développement } \\
\text { économique }\end{array}$ \\
\hline \multirow[b]{2}{*}{$\begin{array}{l}\text { Niveau } \\
\text { de }\end{array}$} & \multirow[b]{2}{*}{ Faible } & F aible & Elevé \\
\hline & & $\begin{array}{l}\text { Pays de } \\
\text { Saint } \\
\text { Flour }\end{array}$ & $\begin{array}{ll}\text { Pays } & \text { Loire } \\
\text { Beauce } & \end{array}$ \\
\hline $\begin{array}{l}\text { capital } \\
\text { social }\end{array}$ & Elevé & $\begin{array}{l}\text { Pays de } \\
D \text { in an }\end{array}$ & Pays du Forez \\
\hline
\end{tabular}

Les enquêtes de terrain (auprès de la population locale et des leaders locaux) avaient pour objectif d'examiner les caractéristiques sociologiques et institutionnelles de ces territoires. Pour cela, nous avons constitué, d'une part, un échantillon de 50 individus par terrain d'étude afin de caractériser le niveau de capital social. Chaque échantillon est représentatif de la population étudiée et a été stratifié : (i) ex ante en fonction du type de zone (i.e. urbain / rural) et de l'âge et (ii) ex post par catégorie socioprofessionnelle. D'autre part, une quinzaine d'entretiens qualitatifs (entretiens semi-directifs) ont été conduits auprès d'acteurs locaux en vue d'appréhender les caractéristiques institutionnelles des terrains étudiés. Nous avons cherché à couvrir la diversité des acteurs en présence. Une première mise en confrontation de ces deux modalités de recueil de l'information nous apporte quelques éclairages significatifs sur les terrains d'application.

63 Les enquêtes réalisées mettent principalement en évidence les résultats suivants.

64 Il existe une analogie entre le comportement des institutionnels et celui des habitants. En effet, dans les territoires où on observe un tissu associatif dense, on constate un fort 
dynamisme des élus locaux. C'est le cas du pays de Dinan et du pays du Forez. Pour le pays de Dinan, la forte tradition de solidarité et d'entraide observée entre les habitants locaux prévaut également dans les dynamiques de coopération entre institutions. Ces dynamiques de relations sociales s'appuient sur une identité territoriale forte. Dans le pays du Forez, on constate une forte organisation locale corrélée à une dynamique de coopération institutionnelle dense (au niveau communal, entre entreprises, etc.). À l'inverse, dans le pays de Saint-Flour/Haute Auvergne et le pays de Loire-Beauce, prévalent plutôt des logiques individualistes ou opportunistes. Dans le pays de LoireBeauce, caractérisé par un certain individualisme et une absence de pratiques coopératives interindividuelles, on observe également des difficultés quant à l'émergence d'actions collectives fédératrices. Les actions collectives sont ponctuelles et répondent à des besoins immédiats. Elles reflètent une certaine logique opportuniste. Au sein du pays de Saint-Flour, le déclin démographique, l'exode des jeunes, le vieillissement continu d'une population cherchant à se protéger du changement (pas de culture d'entreprenariat ni du risque) et peu encline au développement d'une action collective à l'échelle du pays se traduisent par un certain immobilisme local. Dans ce territoire en déprise, les survivances du passé prédominent: absence d'habitude de travail en commun, méfiance, guerre de clochers, difficulté d'insertion des nouveaux venus, etc. Historiquement, en effet, l'entité territoriale de cohésion sociale correspond au hameau. On notera que l'absence de coopération entre les agents (on recense par exemple peu d'initiatives ou de projets collectifs) se reflète également au niveau institutionnel. L'analyse des choix de regroupements intercommunaux montre que ces derniers relèvent davantage de pressions internes et non de l'émergence d'un véritable projet commun porté par les acteurs locaux.

65 Les retombées du jeu relationnel des acteurs locaux se traduisent en termes de développement territorial. Dans le pays du Forez, un fort degré de cohésion sociale sur le territoire est corrélé avec un niveau de développement élevé. Une appréciation de même nature vaut pour le pays de Saint-Flour, pour lequel dynamique de relations sociales et développement territorial sont tous deux faibles.

Les cas intermédiaires sont plus complexes à expliciter. Si les résultats économiques du pays de Dinan - malgré une cohésion sociale forte - restent quelque peu mitigés (-1\% de la croissance de l'emploi par exemple entre 1990 et 1999), une analyse fine de ce territoire permet d'observer une certaine éviction d'effets négatifs (Angeon et Callois, 2004a). Par rapport à d'autres territoires pénalisés par le dynamisme de grandes agglomérations, le pays de Dinan, en périphérie de Rennes et Saint-Malo connaît en réalité une baisse relativement modérée de son niveau d'emploi entre 1990 et 1999 (-1\%). A titre de comparaison, sur la même période, les pays de Dropt (périphérie bordelaise) et de Vierzon (proximité de Bourges) affichent des résultats plus inquiétants (respectivement $-11 \%$ et $-9 \%$ sur la période). Comment expliquer la performance du pays de Loire-Beauce sur le plan du développement territorial malgré un degré de cohésion sociale faible? L'analyse monographique permet là aussi d'affiner la compréhension de ce résultat. Selon les acteurs locaux interrogés, le pays de Loire-Beauce présente la particularité d'être extrêmement ouvert sur l'extérieur. Un indicateur statistique de ce degré d'ouverture tient dans le taux de migration. Au recensement de 1990, 35\% de la population du pays de Loire-Beauce était né en dehors de la région Centre, contre seulement $8 \%$ pour le pays de Dinan par exemple, décrit d'ailleurs comme un territoire à ouverture sur l'extérieure moyenne. Un autre indicateur du degré d'ouverture du pays de Loire-Beauce peut être 
caractérisé par la densité du réseau routier. La distance moyenne de $8 \mathrm{~km}$ à une autoroute quel que soit le point où l'on se situe dans le pays, confère au territoire une bonne accessibilité à l'ensemble des services et des emplois de l'agglomération d'Orléans notamment. Cette ouverture sur l'extérieur expliquerait en partie, selon les acteurs institutionnels interrogés, le fort niveau de développement du territoire.

Ces éléments d'analyse donnent quelques indications sur la nature des relations sociales se déployant sur un territoire comme le pays de Loire-Beauce. Ces liens peuvent s'assimiler à du capital social de type bridging, au sens où ils rendent compte de relations d'échange, temporaires ou ponctuelles, entre acteurs sans qu'il y ait nécessairement antériorité de liens, mais qui sont porteurs d'informations ou de ressources nouvelles et agissant comme des ponts entre territoires.

En résumé, les pays de Loire-Beauce et du Forez ont en commun de raisonner à une échelle territoriale étendue et de présenter une bonne capacité à lancer des actions de coopération opportunes et une bonne gestion de l'ouverture sur l'extérieur. Deux enseignements majeurs peuvent être tirés de nos analyses. D'une part, les territoires à forte composante de bridging sont aussi les plus développés. Il semble donc que les relations sociales de type bridging importent dans les dynamiques de développement territorial. Il convient toutefois de nuancer ce propos, car un fort degré de bridging non articulé avec des liens forts de proximité intra-territoriale risque de fragiliser la cohésion sociale du territoire. C'est le cas du pays de Loire-Beauce où le degré de bridging est certes élevé, mais n'est pas relayé localement par une structure sociale solidaire. Le pays de Dinan parvient quelque peu à contrer les tendances négatives auxquelles il est confronté par l'activation forte d'un capital social de type bonding et linking. Il apparait que les aspects négatifs du capital social sont surtout importants en cas de cohésion sociale forte (manque d'incitation individuelle, effets de collusion entravant les logiques d'action collective notamment) comme cela peut être observé dans le cas du pays de Saint-Flour, marqué par une structure sociale cohésive s'établissant à une échelle spatiale réduite (le hameau).

D'autre part, ces cas d'étude montrent la nécessité d'un équilibre entre les différentes formes de relations sociales dans une dynamique de développement territorial durable. Le cas du pays du Forez en est un bon exemple. Ainsi, contrairement aux idées intuitives et pragmatiques du développement local, un fort degré de cohésion sociale n'est donc pas suffisant pour enclencher une dynamique de développement territorial durable.

Nous argumentons qu'une dynamique de développement territorial durable peut être accompagnée et renforcée de deux façons complémentaires. D'une part, les acteurs locaux peuvent s'appuyer sur les liens de bonding existants et étendre leur espace de relation, en fédérant les initiatives inter-individuelles. D'autre part, l'action publique peut impulser des relations sociales de type bridging, en apportant volontairement un soutien institutionnel au territoire et aux réseaux d'acteurs déjà constitués. Il existe des marges de manœuvre pour augmenter le niveau de capital social, facteur de développement territorial, en agissant sur les ressorts territoriaux.

71 Ainsi, l'activation volontaire du bridging pourrait renforcer la logique de similitude des partenaires. Le partenaire situé sur le territoire pourrait en effet tirer parti de cette ressource relationnelle. Ce faisant, il serait en mesure de développer des relations avec d'autres organisations situées sur le territoire. Cette résultante sociale du bridging n'est autre que du linking. Si les relations de type linking s'entretiennent, on peut penser que sur le long terme elles changent la nature des liens établis, parce qu'elles transforment la 
motivation de la mise en relation ainsi que les propriétés des logiques de similitude qui y président. On obtient alors du bonding. Cela rend compte de la nécessaire articulation entre logique d'appartenance et de similitude dans un processus de développement territorial durable.

En clair, le bridging peut être compris comme un démultiplicateur de capital social, les relations sociales extra-territoriales décuplant les relations intra-territoriales. Dans la construction de projets de développement territorial durable, on peut penser que l'articulation de ces formes d'organisation demande une activation des proximités intra et extra-territoriales entre les acteurs.

Notre analyse des liens sociaux nous permet de considérer de quelle manière les coordinations locales influencent le développement territorial. L'apport de notre contribution tient dans l'articulation de l'économie de proximités et de l'approche du capital social, afin de mieux cerner les mécanismes à l'œuvre dans les processus de développement durable des territoires. Les contributions de ces deux cadres théoriques pour l'analyse des coordinations locales nous ont permis d'appréhender l'importance des dynamiques sociales dans ces processus de développement. Plus largement, notre conception du capital social dans sa capacité à impulser des dynamiques de construction territoriale s'apparente à la notion de ressources spécifiques (Colletis et Pecqueur, 1993, 2004).

74 Nos études monographiques suggèrent que ce ne sont pas seulement les relations de proximité forte qui importent dans les logiques de développement territorial mais aussi, et parfois de manière prédominante, les relations de distance. Ces liens extra-territoriaux et non nécessairement activés rompent partiellement avec les caractéristiques organisationnelles et institutionnelles que l'économie de proximités prête aux coordinations efficaces. Les processus de développement reposent ainsi sur une matrice de proximité dans laquelle l'importance des liens faibles et leur capacité à renforcer et stimuler les liens forts peuvent se révéler prépondérantes. Les trois formes de capital social s'imbriquent et peuvent se transformer. En termes d'intervention publique territoriale, on peut insuffler de nouvelles dynamiques, en favorisant l'établissement des liens sur des ressorts territoriaux activables.

75 L'articulation entre l'économie de proximités et l'approche du capital social nous a ainsi semblé pertinente pour la compréhension des déterminants de la performance économique locale. Leur combinaison avec une approche spatiale ouvre la voie à une meilleure compréhension et à de nouvelles possibilités d'activation des ressorts territoriaux. Elles nous paraissent à ce titre toutes trois essentielles à une théorisation des mécanismes de développement durable des territoires.

\section{BIBLIOGRAPHIE}

Adler P., Kon S. W., 1999, “Social capital: the good, the bad, and the ugly”, Research Paper, Dept. of management and organization, University of Southern California, Los Angeles. 
Angeon V., Callois J.-M., 2004, De l'importance des facteurs sociaux dans le développement, 1ères journées du GRES, Le concept de développement en débat, Université Montesquieu-Bordeaux IV, 16 et 17 septembre 2004.

Angeon V., CAlloisS J.-M., 2004b, Fondements théoriques du développement local : quels apports de la théorie du capital social et de l'économie de proximité ? The 4th Congress on Proximity Economics, Proximity, Networks and Co-ordination, Marseille, June 17 - 18.

Angeon V., CAllois. (Dir.), 2005, Rôle des coordinations locales dans le développement durable des territoires, Rapport de recherche commandité par le MEDD et le PUCA, juillet 2005.

Arrow K., 1974, The limits of organizations, W. W. Norton \& Company, New York.

Bellet M., Colletis G., Lung Y., (Eds), 1993, « Economie des proximités », Revue d'économie régionale et urbaine, numéro spécial $n^{\circ 3}$, pp 357-606.

Bellet M., Kirat T., Largeron C., (Eds), 1998, Approches multiformes de la proximité, éditions Hermès, Paris.

Barrere C., Barthelemy D., OlagnonH., Nieddu M., Vivien F.-D., 2001, Patrimoine, approches croisées, Ecole doctorale des sciences juridiques, économiques et gestion, Université de Reims, Cahier $n^{\circ} 2$, mars.

Billaudot B., 2004, Proximité, réseaux et coordination industrielle. Quelle articulation conceptuelle?, The 4th Congress on Proximity Economics, Proximity, Networks and Co-ordination, Marseille, June 17-18.

Bourdieu P., 1980, "Le capital social : notes provisoires". In Actes de la recherche en sciences sociales, volume 31, pp. 2-3

Bourdieu P., 1986, "The forms of capital", in Richardson J.G., (Ed.), Handbook of theory and research for the sociology of education, New York, pp. 241-258.

Bourdieu P., 1987, "What makes a social class? On the theoretical and practical existence of groups”, Berkeley Journal of Sociology, 32, pp. 1-17.

Calame P., 1991, « Projets de ville, projets de vie, esquisse d'une théorie de l'action collective », Actes, $\mathrm{n}^{\circ} 5$, Octobre.

Calois J.-M., 2004, « Capital social et développement économique local : pour une application aux espaces ruraux français ", Revue d'Economie Régionale et Urbaine, 4, pp. 551-578.

Coleman J. S., 1988, "Social capital in the creation of human capital”, American journal of sociology, vol. 94, pp. S95-S120.

Coleman J. S., 1990, Foundations of social theory, Harvard University Press, Cambridge.

Colletis G., Pecqueur B., 1993, «Intégration des espaces et quasi-intégration des firmes : vers de nouvelles rencontres productives ", Revue d'Economie Régionale et Urbaine, n³, pp. 489-508.

Colletis G., Pecqueur B., 2004, Révélation de ressources spécifiques et coordination située, The 4th Congress on Proximity Economics, Proximity, Networks and Co-ordination, Marseille, June 17 - 18.

Collier P., 1998, "Social capital and poverty", Social capital Initiative Working paper, World Bank, Social Development Department, Washington, D.C. Processed.

Debarbieux B., 2003, « Neuf enjeux de l'iconographie de projet et de prospective de territoire », in Debarbieux B., Lardon S. (dir.) Les figures du projet territorial, Editions de l'Aube, Datar, Bibliothèque des territoires, pp 13-36. 
Deffontaines J.-P., Marcelpoil E. et Moquay P., 2001, « Le développement territorial : une diversité d'interprétations ", in Lardon S., Maurel P., Piveteau V. (éds), Représentations spatiales et développement territorial. Bilan d'expériences et perspectives méthodologiques, Paris, Hermès, pp. 39-56. Dupuy., Torre A., 1998, «Liens de proximité et relations de confiance : le cas des regroupements localisés de producteurs dans le domaine alimentaire » in Bellet M., Kirat T., Largeron C., (Coord), 1998, Approches multiformes de la proximité, Eds. Hermès, Paris, pp. 175-192.

Fukuyma F., 1999, « Social capital and civil society », IMF Conference on second generation reforms, $12 \mathrm{p}$.

Gilly J.-P., Torre A., (Dir.), 2000, Dynamiques de proximité, Ed. L'Harmattan. Coll. Emploi, industrie et territoire, Paris.

Granoveter M., 1973, “The strength of weak ties”, American Journal of Sociology, 78, 6, pp. 1360-1380.

Greffe X., 2002, Le développement local, Bibliothèque des territoires, Ed. de l'aube DATAR, Paris. Grosetti M., 1998, « La proximité en sociologie : une réflexion à partir des systèmes locaux d'innovation ", in Bellet M., Kirat T., Largeron C., (Coord). 1998, Approches multiformes de la proximité, Eds. Hermès, Paris, pp. 83-100

KnackS., Keefer P., 1997, “Does Social Capital Have an Economic Payoff? A Cross-Country Investigation”, Quarterly Journal of Economics, vol. 112, n 4, pp. 1251-1288.

Krishna A., 2001, "Moving from the Stock of Social Capital to the Flow of Benefits: The Role of Agency", World Development, vol. 29, n 6, pp. 925-943.

Krishna A., 2000, “Creating and harnessing social capital”, in P. Dasgupta et I. Serageldin (Eds.), Social Capital: a multifaced perspective, World Bank, Washington, DC., pp. 71-93.

Laganier R., Villalba B., Zuindeau B., 2002, « Le développement durable face au territoire : éléments pour une recherche pluridisciplinaire », Revue Développement durable et Territoires, Dossier 1 : Approches territoriales du Développement Durable.

Lardon S., Piveteau V., Warrot G., Mahe C., 2003, « Entre territoires institutionnels et territoires fonctionnels : l'organisation spatiale des territoires de projet », Colloque Territoires institutionnels, territoires fonctionnels, Macon 25-27 Septembre 2003, (à paraître).

Le Berre, M., 1995, Territoire, in A. Bailly, R. Ferras, D. Pumain, Encyclopédie de Géographie, éditions Economica, Paris.

Levesque M., 2000, Le capital social comme forme sociale de capital : reconstruction d'un quasiconcept et l'application à l'analyse de la sortie de l'aide sociale, Université de Montréal, août.

Ln N., 1995, «Les ressources sociales : une théorie du capital social », Revue Française de sociologie, 36 , pp. 685-704.

Lin N., 2001, "Building a theory of social capital" in N. Lin, K. Cook and R. S. Burt (eds.), Social capital: theory and research, Aldine-de Gruyter, New York.

MoquayY P., Lardon S., MarcelpoilL E., Piveteau V., 2005, « Contribution des représentations spatiales à la proximité institutionnelle ", in A. Torre et M. Filippi (eds) Proximités et Changements socio-économiques dans les mondes rurau, Editions de l'INRA, collection Science en Marche,14 p.

Pecqueur B., Zimmerman J.-B., Dir., 2004, Economie de proximités, Ed. Hermès, Paris.

Perroux F., 1964, L'économie du XXe siècle, PUF, Paris. 
Piveteau V., Lardon S., 2002, « Chorèmes et diagnostics de territoire : une expérience de formation », Mappemonde 68, 4, pp 1-6.

Ponthieux S. (2003), «Que faire du "social capital ? ", Série des documents de travail de la Direction des statistiques démographiques et sociales, INSEE, ${ }^{\circ} \mathrm{F} 0306$.

Portes A, 1998, "Social capital: its origins and applications in modern sociology", Annual Review of Sociology, 24, pp. 1-24.

PortesA, Landolt P., 1996, “The downside of social capital”, American Prospect, 7, 26, pp. 18-21.

Putnam R. D., 1993, Making democracy work: civic traditions in modern Italy, Princeton university press, Princeton.

Putnam R., 1995, “Tuning in, tuning out: the strange disappearance of social capital in America", Political Science and Politics, December, 28 (4), pp. 664-683.

Rallet A. (2002), "L'économie de proximité. Propos d'étape", in TORRE A. (éd.), Etudes et Recherches sur les Systèmes Agraires et le Développement, n³3, Le local à l'épreuve de l'économie spatiale, pp. 11-25.

Sandefur R., Lauman E., 1998, “A paradigm for social capital”, Rationality and Society, 10, pp. 681-501.

Torre A., Caron A., 2002, « Conflits d'usage et de voisinage dans les espaces ruraux », Sciences de la Société, $\mathrm{n}^{\circ} 57$, pp.95-113.

Uphoff N., 2000, "Understanding social capital: learning from the analysis and experience of participation", in P. Dasgupta, I. Serageldin (Eds), Social capital: a multifaced perspective, Washington, D.C., pp. 215-249.

Woolcok M., 2001, « La place du capital social dans la compréhension des résultats sociaux et économiques », DRHC/OCDE.

Zimerman J.-B, Gilly J.-P, Perrat J., Pecqueur B., Rychen F., 1998, Construction territoriale et dynamiques productives, Etude pour le commissariat général du plan, décembre, $261 \mathrm{p}$.

Zuindeau B., Ed., 2000, Développement durable et territoire, Presses universitaires de Septentrion, Villeneuve d'Asq.

\section{NOTES}

1. Encore faut-il s'interroger sur l'applicabilité de ces principes élaborés originellement à l'échelle globale (Zuindeau, 1996).

2. Pour Laganier, Villalba et Zuindeau (2002), le DD repose sur le double principe d'interdépendance et d'équité. L'interdépendance porte sur trois registres : temporel, spatial et caractérise également "les domaines de la vie sociale et de son environnement naturel".

3. Les nouvelles formes de recomposition territoriale et leurs outils (pays, intercommunalité par exemple) constituent des cadres pour l'établissement d'un développement territorial durable.

4. Cette loi dite Voynet (25 juin 1999) propose de nouvelles modalités d'organisation territoriale pour mettre en place les conditions d'un développement durable. Elle institue les « pays ». 
5. Cette loi dite Chevènement (12 juillet 1999) offre un cadre de coopération qui impulse de nouvelles logiques de développement territorial.

6. Cette loi dite Gayssot (13 décembre 2000) actualise les dispositions de la loi d'orientation foncière (décembre 1967) en s'appuyant sur deux outils : le schéma de cohérence territoriale (SCOT) et le plan local d'urbanisme (PLU). Elle implique que soient entrepris des choix cohérents de déplacement, d'implantation de zones d'activités, de maîtrise de l'étalement urbain, de protection des espaces fragiles, etc.

7. On notera, à ce titre, que les « pays » sont des « Agendas 21 locaux ».

8. Cette section s'inspire d'une réflexion plus large menée dans le cadre d'un programme de recherche financé par le Ministère de l'Ecologie et du Développement Durable et le Ministère de l'équipement dont nous reprenons les principaux résultats dans la dernière partie du texte.

9. Les premiers travaux recensés dans la littérature remontent à Weber et Simmel.

10. Divers travaux se démarquent quant à l'identification des formes de capital social. On citera en particulier les analyses de Collier (1998) et de Uphoff (2000). Collier (1998) distingue capital social gouvernemental et civil. Le premier fait référence aux institutions ou organisations mises en place par l'Etat ou le secteur public alors que le second fait référence aux institutions ou organisations émanant de la société. Uphoff (2000) différencie le capital social structurel (entendu comme l'ensemble des institutions visibles) du capital social cognitif (ou subjectif c'est-à-dire qui renvoie aux processus mentaux des individus). En clair, le premier désigne les structures dans lesquelles agissent les agents (i.e. les organisations) alors que le second fait référence aux valeurs, normes, croyances etc. qui prédisposent les agents à la coopération. Pour désigner cette même distinction de nature sur le capital social, Krishna (2000) parle respectivement de capital social institutionnel et relationnel.

11. Cependant, pour Bourdieu $(1980,1987)$ le capital social n'a pas de contenu propre, c'est un multiplicateur de capital. Il est en cela second par rapport aux autres formes de capitaux. C'est un "méta- capital".

12. À titre d'exemple, un étudiant peut financer ses études par le biais de son réseau social. Il aura ainsi accès à une partie du capital économique contenu dans ses relations sociales. Mais ce même individu peut ne recevoir aucun soutien financier si les membres de ce réseau ne disposent pas de capital économique.

13. Si l'on reprend l'exemple de l'étudiant, les configurations peuvent être différenciées. Un individu peut financer ses études par le biais de son réseau social alors qu'un autre membre du même réseau n'aura pas nécessairement accès au même niveau de ressource financière.

14. « La proximité géographique de configuration découle de l'appartenance au même type de configuration spatiale. La configuration spatiale renvoie en première analyse à des types génériques de localisation, dont les caractéristiques peuvent être comparées ; elle reflète en complément l'adaptation spécifique des pratiques à cet environnement. Elle est ainsi le résultat de la combinaison d'actions des hommes pour maîtriser les processus naturels et développer les activités en un lieu donné. On peut dès lors constater et expliquer des rapprochements entre situations a priori distantes, mais qui en fait relèvent des mêmes logiques ».

15. cette forme de proximité prédispose à une logique de relation à distance.

16. La capacité d'entretien du lien varie selon un nombre important de critères (fonction eux-mêmes des agents et de leur habitudes relationnelles) qui rendent impossible de définir la vitesse avec laquelle se déprécie le capital social. 
17. Ces éléments d'appréciation sont répertoriés en annexe.

18. Cf. en annexe la présentation du questionnaire.

\section{RÉSUMÉS}

Notre article s'intéresse au rôle des relations sociales dans les processus de développement territorial durable. C'est à une analyse de la coordination locale, qui prend en compte l'importance des facteurs non marchands, que nous invite l'exploration théorique de ces mécanismes de développement. Nous retenons, pour ce faire, les approches du capital social et de la proximité. Après avoir étudié la manière dont les coordinations locales influent sur les dynamiques de développement territorial, nous montrons, dans la deuxième partie de ce texte, la complémentarité de ces deux champs théoriques. Dans la dernière partie de ce texte, nous nous intéressons à une caractérisation spatiale des liens sociaux. Nous mobilisons les trois registres de la proximité pour qualifier la traduction spatiale des relations sociales et comprendre la manière dont elles évoluent dans la perspective d'un développement territorial durable.

From social links to territorial and sustainable development. How does proximity matter in that process?

This article points out the role of social relationships on territorial and sustainable development process. Our analysis of local co-ordination - which underlines the importance of non market factors - originates in the economics of proximity and the theories of social capital. We first state how social relationships influence local development. The second part of this paper stresses that the two approaches complement each other. We show how the articulation between the two theoretical frameworks is fundamental to properly comprehend the social determinants of territorial development dynamics. The third part of the text mainly focuses on the spatial impacts of social relationships. We propose an analytical grid to identify and qualify them in a sustainable development process.

\section{INDEX}

Mots-clés : proximité, développement territorial, capital social, configurations spatiales

Keywords : proximity, territorial development, social capital, spatial configurations

\section{AUTEURS}

\section{VALÉRIE ANGEON}

Valérie Angeon est Maître de conférences en économie à l'ENGREF depuis 2002. Ses travaux portent sur l'analyse des logiques d'action collective pour le développement territorial.UMR METAFORT POP'TER ENGREF, 24 Avenue des landais, 63170 AUBIERE, Email : angeon@engref.fr Tel : 04734407 19, Fax : 0473440700 


\section{PATRICK CARON}

Patrick Caron est géographe et chercheur au CIRAD depuis 1988. Il s'intéresse à l'analyse et à l'accompagnement des dynamiques territoriales dans les régions tropicales.

CIRAD,UMR TETIS, TA 60/15, 73, Avenue Jean-François Breton, 34398 MONTPELLIER

Cedex 5, E-mail : patrick.caron@cirad.fr, Tel : 046759 38 49, Fax : 0467614414

\section{SYLVIE LARDON}

Sylvie Lardon est Directrice de Recherche à l'INRA-SAD et Professeure extérieure à

l'ENGREF depuis 2001. Biométricienne, elle appréhende les dynamiques territoriales par le recours aux configurations spatiales. UMR METAFORT - POP'TER ENGREF, 24 Avenue des landais, 63170 AUBIERE, E-mail : lardon@engref.fr, Tel : 04734407 21, Fax : 047344 0700 